\title{
Implementation of depression screening in antenatal clinics through tablet computers: results of a feasibility study
}

José S. Marcano-Belisario ${ }^{1 *}$, Ajay K. Gupta ${ }^{2}$, John O’Donoghue ${ }^{3}$, Paul Ramchandani ${ }^{4}$, Cecily Morrison ${ }^{5}$ and Josip Car ${ }^{6}$

\begin{abstract}
Background: Mobile devices may facilitate depression screening in the waiting area of antenatal clinics. This can present implementation challenges, of which we focused on survey layout and technology deployment.

Methods: We assessed the feasibility of using tablet computers to administer a socio-demographic survey, the Whooley questions and the Edinburgh Postnatal Depression Scale (EPDS) to 530 pregnant women attending National Health Service (NHS) antenatal clinics across England. We randomised participants to one of two layout versions of these surveys: (i) a scrolling layout where each survey was presented on a single screen; or (ii) a paging layout where only one question appeared on the screen at any given time.

Results: Overall, $85.10 \%$ of eligible pregnant women agreed to take part. Of these, $90.95 \%$ completed the study procedures. Approximately $23 \%$ of participants answered Yes to at least one Whooley question, and approximately $13 \%$ of them scored 10 points of more on the EPDS. We observed no association between survey layout and the responses given to the Whooley questions, the median EPDS scores, the number of participants at increased risk of self-harm, and the number of participants asking for technical assistance. However, we observed a difference in the number of participants at each EPDS scoring interval $(p=0.008)$, which provide an indication of a woman's risk of depression. A scrolling layout resulted in faster completion times (median $=4$ min $46 \mathrm{~s}$ ) than a paging layout (median $=5 \mathrm{~min} 33 \mathrm{~s}$ ) ( $p=0.024)$. However, the clinical significance of this difference (47.5 s) is yet to be determined.

Conclusions: Tablet computers can be used for depression screening in the waiting area of antenatal clinics. This requires the careful consideration of clinical workflows, and technology-related issues such as connectivity and security. An association between survey layout and EPDS scoring intervals needs to be explored further to determine if it corresponds to a survey layout effect. Future research needs to evaluate the effect of this type of antenatal depression screening on clinical outcomes and clinic workflows.
\end{abstract}

Trial registration: This study was registered in ClinicalTrials.gov under the identifier NCT02516982 on 20 July 2015.

Keywords: Antenatal depression, Mental health, Edinburgh Postnatal Depression Scale (EPDS), Whooley questions, Population screening, Patient self-report, Mobile health (mHealth), Apple ${ }^{\circledR} \mathrm{iPad}{ }^{\oplus}$, Tablet computers, Survey layout

\footnotetext{
* Correspondence: jose.marcano-belisario10@imperial.ac.uk

${ }^{1}$ Global eHealth Unit, Department of Primary Care and Public Health, School

of Public Health, Imperial College London, London, UK

Full list of author information is available at the end of the article
} 


\section{Background}

The role of mobile technology for the assessment and screening of mental health disorders has received much attention in recent years [1-5]. Devices such as smartphones and tablet computers may facilitate the practical implementation of mental health population screening programmes in clinical settings. For patients, these devices could make the completion of screening scales more convenient, while increasing the perceived sense of confidentiality. The potential advantage for clinicians includes the rapid and convenient access to patients' results. As recent evidence suggests that using mobile devices to administer validated screening scales does not affect their overall data equivalence (when compared to traditional delivery modes such as paper) [6], it is timely to explore the implementation of mobile-based screening.

Mobile-based screening programmes could be particularly beneficial for maternal mental health, especially antenatal depression. This condition is one of the most common during pregnancy, affecting between $7 \%$ and $12 \%$ of pregnant women $[7,8]$. If left untreated, antenatal depression can have severe and long-lasting consequences for mothers, and their children and families [915]. Moreover, the antenatal period offers an opportunity of frequent interactions between pregnant women and their healthcare providers. Screening scales may be administered during this cycle of encounters as recommended by clinical guidelines [16].

Research has shown that the use of screening scales in the waiting area of clinical facilities can work well [17]. Patients who complete surveys whilst waiting for their appointment tend to report reduced frustration levels. Gathering data beforehand could also trigger meaningful discussions during the consultation that patients may have trouble raising directly [17]. Lastly, this could facilitate the monitoring of disease progression and treatment response, and help to ensure that important issues are not overlooked by the clinical team [17].

However, the use of mobile technology for screening in clinical settings presents implementation challenges of which we focus on two: survey design and deployment. Survey layout can influence respondents' behaviour, a phenomenon known as survey layout effect [1820]. Scrolling layouts (i.e., presenting all questions on a single screen) are thought to result in higher subjective ratings, lower breakoff and item non-response rates, fewer technical problems, and faster completion times [18] than paging layouts (i.e., presenting one question per screen). Although these findings relate to volunteer activities surveys, they could have clinical implications.

Depending on its size and direction, a survey layout effect on the subjective ratings given to clinical scales could result in changes to overall clinical assessments for reasons other than the construct under assessment.
This could result in patients needing to undergo unnecessary diagnostic pathways due to false positive results, or in diagnostic delays due to false negative results. For clinical teams, there could be implications in terms of increased workload due to false positive results, or liability due to false negatives.

The findings from the survey methodology literature [18] also raise practical implications for the deployment of mobile technology in clinical settings. The potential for added workload for clinical staff, training and capacity needs, location and privacy of screening, network issues, and responsibility for technology are some of the perceived barriers to the success of mobile-based antenatal depression screening $[5,21]$. Indicators from survey methodology studies (e.g., completion times, breakoff rates, technical problems) [18] may allow practitioners to capture useful information about the real impact of these barriers, giving opportunity to address them.

The aim of this study was to assess the feasibility of using tablet computers $\left(\right.$ Apple $^{\oplus} \mathrm{iPads}^{\odot}$ ) in the waiting area of antenatal clinics for implementing the recommendations of the National Institute for Health and Care Excellence (NICE) guidelines for recognising antenatal depression [16]. Our main outcomes were participants' responses to the Whooley questions and the EPDS, and whether these differed from prevalence depression rates reported in the literature. In addition, we chose completion time and the number of participants requesting technical assistance as indicators of the impact that the deployment of mobile technology could have on the workload and schedules of clinics. Furthermore, we explored if there were any differences between two survey layouts across all study outcomes. We also assessed smartphone and tablet computer ownership amongst our participants. The latter can provide an indication of participants' familiarity with mobile technology, and of the potential reach of future mobile health interventions. Breakoff rates were interpreted in relation to the incompatibility between research procedures and clinic schedules. Lastly, we report the deployment challenges that we identified during this feasibility study. We did not assess item non-response rates, as validation procedures did not allow participants to leave questions unanswered.

\section{Methods}

For the protocol of this feasibility study see MarcanoBelisario and colleagues [22].

We assessed the feasibility of using tablet computers to administer a socio-demographic survey, the Whooley questions and the Edinburgh Postnatal Depression Scale (EPDS) to 530 pregnant women attending antenatal clinics in National Health Service (NHS) facilities across England. We used a randomised controlled study design 
to allocate participants to one of two layout versions of these surveys: a paging layout or a scrolling layout.

\section{Participants and participant recruitment}

We recruited adult (18 years or older) pregnant women of any gestational age, pregnancy history and parity. We excluded women who had been diagnosed with depression or generalised anxiety disorder in the past 12 months (from the moment they were approached by the research team), or who were currently receiving treatment for any of these disorders. We also excluded women who did not feel comfortable reading and writing in English.

Recruitment took place between October 2015 and May 2016 using an opportunistic approach. Pregnant women attending antenatal clinics in any of the participating centres (i.e., antenatal clinics in general practices, community services and hospitals) were approached in the waiting area by a research midwife or a clinical studies officer (CSO) with information about the study and a copy of the participant information leaflet (PIL). Those consenting to take part in the study were then asked to complete the study procedures in the clinic's waiting area before their appointment.

As shown in Fig. 1, 699 eligible women were approached in the waiting area of participating clinics.
Of these, 632 agreed to take part in the study and initiated the consent process. In relation to the total number of eligible participants $(N=743)$, our recruitment rate was $85.10 \%$. However, 44 eligible women were called in before they could be approached by a research midwife or CSO. Therefore, our recruitment rate in relation to the total number of eligible women who were approached $(N=699)$ was $90.41 \%$.

Of the 632 women who initiated the consent process, 35 were called in before they could complete the informed consent form. Therefore, 597 participants were recruited into the study. Of these, 54 were called in (27 participants from the Scrolling group and 27 participants from the Paging group) before completing the surveys. Therefore, our completion rate was $90.95 \%$ (543 participants out of the 597 who were initially randomised).

\section{Technology and materials}

We designed the surveys in Snap survey software [23], and administered them through Snap ${ }^{\circ}$ Mobile app (versions 4.0.30 to 4.0.32) for Apple ${ }^{\oplus}$ iPhone Operating System $\left(\mathrm{iOS}^{\mathrm{TM}}\right)$ [24] running on Apple ${ }^{\circ} \mathrm{iPad}_{\text {Air }}{ }^{\circ}$ and Apple $^{\circ}$ iPad mini ${ }^{\mathrm{TM}}$ tablet computers. Responses were stored in Snap ${ }^{\circ}$ WebHost [25]. These solutions supported the required survey layouts and offline data collection. The latter being important due to the variability

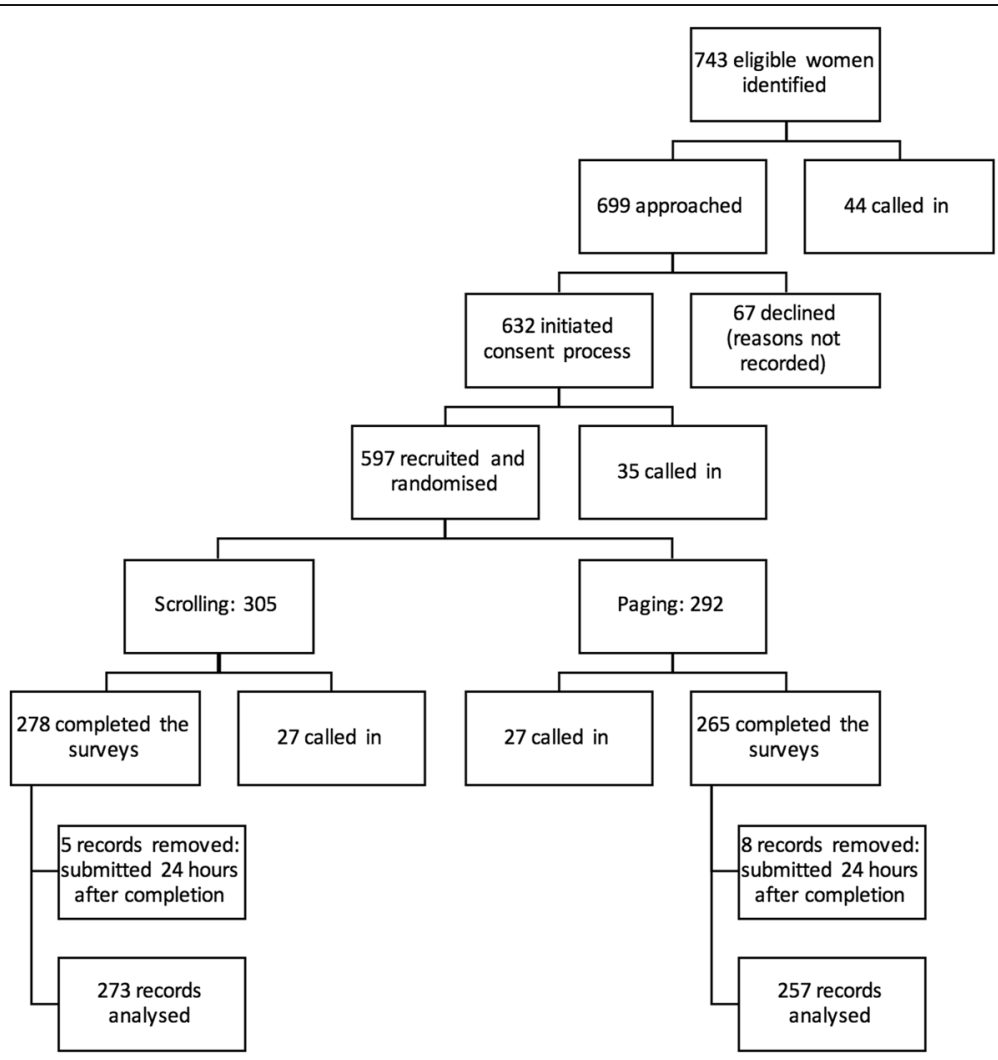

Fig. 1 Study flow 
in internet access across NHS facilities. We originally intended to use Apple ${ }^{\oplus}$ iPad Air ${ }^{\circ}$ tablet computers to control for screen size; due to limited resources however, we had to use Apple ${ }^{\circ}$ iPad mini ${ }^{\mathrm{TM}}$ tablet computers in some sites.

\section{Surveys}

We developed an 11-question socio-demographic survey to assess participants' age group, ethnicity, relationship status, employment status, level of education, smartphone and tablet ownership, pregnancy history and parity, pregnancy trimester, and previous history of depression (Additional file 1).

Following NICE recommendations [16], we used the Whooley questions and the EPDS. The former is a 2item survey evaluating the presence of depressed mood and anhedonia over the past month [26] (Additional file 2). An affirmative answer to either question should be followed up by further assessment using a validated screening tool such as the EPDS [16].

The EPDS is a 10-item instrument used to screen for antenatal or postnatal depression in community and clinical settings (see Additional file 3) [27]. This instrument assesses feelings of guilt, sleep disturbance, anhedonia (i.e., the inability to derive pleasure from activities considered to be pleasurable) and thoughts of self-harm that have been present for the past 7 days. Each question is scored on a 4-point scale ranging from 0 to 3 points. Overall scores are generated by the sum of these responses. Scores between 10 and 12 points suggest increased risk for depression, and scores of 13 points or more suggest that the diagnostic criteria for major depression have been met [28]. In these cases, patients should undergo formal diagnostic assessment [16]. Scores of 1 point or more on question 10 of the EPDS (regardless of the overall score), also require further assessment as this question deals with self-harm.

Although NICE guidelines recommend using a twostaged approach where the EPDS is administered only if there is an affirmative answer to at least one Whooley question, all the participants in this study completed the EPDS regardless of their answers to the Whooley questions.

\section{Scrolling layout}

In this type of survey layout, each survey was presented on a single screen (see Fig. 2). Therefore, participants had to scroll vertically in order to answer all the questions. Participants were allowed to navigate vertically and between surveys, and to modify their answers to any questions before submitting their answers. After pressing the submit button, they were asked to return the tablet computer to the research midwife or CSO who was then presented with a summary of the participant's results. This information was used to complete a summary letter, which was given to a midwife or consultant in time for the consultation. Validation rules were applied with the app, which prevented participants from leaving any questions unanswered.

\section{Paging layout}

The study procedures for this type of survey layout were identical as those for the Scrolling Layout. The difference was that only one question was displayed on the screen at any given time (see Fig. 3). Participants were allowed to navigate between questions and to modify their answers before pressing the submit button.

\section{Study procedures Randomisation}

We used block randomisation (with blocks of 4) to allocate participants to one of the two manipulations of the survey layout: paging or scrolling. For each participating site, we generated an independent sequence using Stata V.13.0 [29]. These sequences were printed and placed inside opaque, numbered envelopes that were then sealed and distributed. Research midwives and CSOs were not involved in generating random sequences. Envelopes were used sequentially and opened once a participant had completed the informed consent form. The contents of the envelope informed the research midwife or CSO regarding which version of the surveys they should activate; at this point, they handed over the tablet computer to the participant. If a participant was called in before completing the surveys, her record on the tablet was cancelled and the content of that randomisation envelope discarded.

\section{Statistical analyses}

We compared the two survey layouts on participants' responses to the Whooley questions and the EPDS separately. To this end, we compared the proportion of participants answering Yes to at least one Whooley question between the Scrolling and Paging groups. We also compared these groups in relation to the median EPDS scores, the proportion of participants scoring at each interval of the EPDS (i.e., 0 to 9 points, 10 to 12 points, and 13 points or more), and the proportion of participants scoring 1 point or more on question 10 of the EPDS.

Moreover, we presented the overall median completion time (seconds) across all participants and evaluated differences on this outcome between the Scrolling and $\mathrm{Pa}$ ging groups. We also compared the proportion of participants requesting technical assistance between these groups. In addition, we measured smartphone and tablet ownership across all participants.

We used a chi-squared test to compare proportions of participants, and the Wilcoxon rank-sum test as the EPDS scores and the completion times were not normally distributed.

We conducted all our analyses using $\mathrm{R}$ version 3.3.1 [30]. 


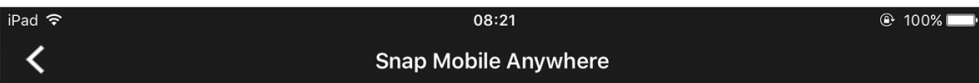

Section 3: Edinburgh Postnatal Depression Scale

As you are pregnant, we would like to know how you are feeling.

Please check the answer that comes closest to how you have felt in the past 7 days, not just how you feel today.

Q3.1 In the past 7 days, I have been able to laugh and see the funny side of things:

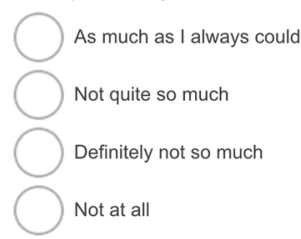

Q3.2 In the past 7 days, I have looked forward with enjoyment to things:

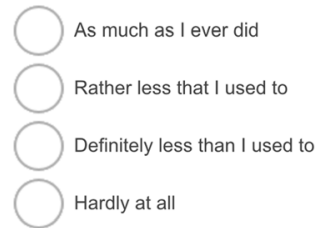

Q3.3 In the past 7 days, I have blamed myself unnecessarily when things went wrong:

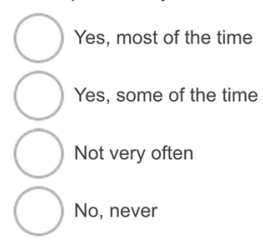

Q3.4 In the past 7 days, I have been anxious or worried for no good reason:

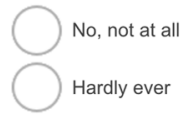

Fig. 2 Scrolling layout - Screenshot

\section{Results}

Of 597 pregnant women who consented to participate, 543 completed the study procedures (see Fig. 1). However, we removed 13 records as they were submitted more than $24 \mathrm{~h}$ after completion. Therefore, we included 530 records in our analysis: 273 from the Scrolling group and 257 from the Paging group. Participants were recruited from 14 localities across England (Blackburn, Bolton, Burton, Bury, East Midlands, London, Huntingdon, North East, Oldham, Rochdale, Shrewsbury, Stoke-on-Trent, Telford, and Wigan). Table 1 provides a summary of the socio-demographic characteristics of our sample.

\section{Participants' responses to recommended depression screening scales Whooley questions}

Overall, 122 (23.02\%) participants answered Yes to at least one Whooley question and 408 (76.98\%) participants answered No to both questions (Table 2).
We performed a chi-squared test of independence to examine the relation between participants' answers to the Whooley questions (i.e., the number of participants answering Yes to one or both Whooley questions and those answering No to both questions) and survey layout (i.e., Scrolling and Paging) (Table 2). The relation between these variables was non-significant, $\chi^{2}(1, N=$ 530) $=0.0049718, p=0.944$.

\section{Edinburgh postnatal depression scale}

Table 3 shows the mean and median EPDS scores for all participants, as well as by survey layout allocation. We compared the median EPDS scores between both survey layouts using a Wilcoxon rank-sum test and found no difference between them: $\mathrm{W}=35755, p=0.700$.

Table 4 shows the overall number of participants at each EPDS interval, as well as by survey layout allocation. We performed a chi-squared test of independence to examine the relation between EPDS scoring intervals and survey 


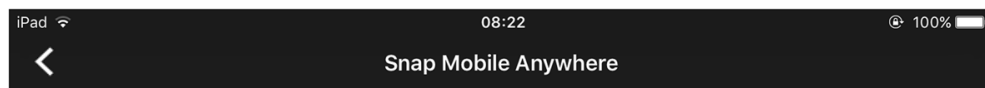

Q3.1 In the past 7 days, I have been able to laugh and see the funny side of things:

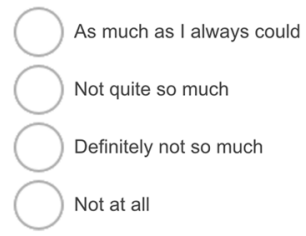

\section{$\leftarrow$ Back Next $\rightarrow$}

Fig. 3 Paging layout - Screenshot

layout (Table 4). The relation between these variables was significant, $\chi^{2}(2, N=530)=9.6779, p=0.008$.

Table 5 shows the overall number of participants by their score on question 10 of the EPDS, as well as by survey layout allocation. We performed a chi-squared test of independence to examine the relation between participants' scores on question 10 of the EPDS and survey layout (i.e., Scrolling and Paging) (Table 5). The relation between these variables was non-significant $\chi^{2}(1$, $N=530)=0.010247, p=0.919$.

\section{Completion time and requests for technical assistance}

For our calculations concerning completion time, we excluded participants who requested technical help from the local research team during survey completion. Therefore, we included data from 495 participants: 254 from the Scrolling group and 241 participants from the Paging group.

Overall, $75 \%$ of participants completed the study procedures in $7 \mathrm{~min} 16 \mathrm{~s}$ or less, and half of them in $5 \mathrm{~min} 9 \mathrm{~s}$ or less. These completion times included the baseline demographic data survey, and are thus an overestimation of the real duration of the screening process (i.e., Whooley questions and EPDS). To give a sense of the overestimation, we trialled the Whooley questions and the EPDS with colleagues, which yielded estimates of 2 min $28 \mathrm{~s}$. Using a Wilcoxon rank-sum test, we observed that participants in the Scrolling group were faster at completing the surveys $(\mathrm{Mdn}=285.5 \mathrm{~s})$ than participants in the Paging group $(\mathrm{Mdn}=333 \mathrm{~s}): \mathrm{W}=27014, p=0.024$.

Overall, 35 participants made requests for technical assistance. We performed a chi-squared test of independence to explore the relation between the number of participants asking for technical assistance and survey layout (i.e., Scrolling and Paging) (Table 6). The relation between these variables was non-significant $\chi^{2}(1, N=$ 530) $=0.027251, p=0.869$.
No participant made more than one request for technical assistance. In addition, Table 7 provides a summary of the types of requests made.

\section{Smartphone and tablet computer ownership}

From the 530 participants included in the analysis, 518 $(97.74 \%)$ reported owning a smartphone and 435 $(82.10 \%)$ reported owning a tablet computer.

\section{Discussion}

Participants' answers to the Whooley questions and the EPDS

In this study we assessed the feasibility of using tablet computers in the waiting area of antenatal clinics for implementing NICE recommendations for the recognition of antenatal depression. Concerning participants' responses to the depression screening scales, we observed that approximately $23 \%$ of participants answered Yes to at least one Whooley question. In addition, approximately $13 \%$ of participants scored 10 points or more on the EPDS. These are screening scales and, as such, do not provide a diagnosis of depression. However, these figures are within the expected prevalence rates of antenatal depression. Therefore, our findings suggest that depression screening using tablet computers may not result in a disproportionate number of patients screening positive.

Moreover, we compared two survey layouts (i.e., Scrolling vs Paging) on participants' responses to the Whooley questions and the EPDS. We observed no relation between survey layout (i.e., Scrolling and Paging) and participants' answers to the Whooley questions, participants' median EPDS scores, and participants' answers to question 10 of the EPDS (which deals with thoughts of self-harm). However, we found a significant relationship between survey layout and EPDS scoring thresholds.

The current study design does not allow us to determine if these findings are the result of a survey layout effect or a difference in the proportion of participants with 
Table 1 Summary of participants' socio-demographic characteristics

\begin{tabular}{|c|c|c|c|}
\hline & & $\begin{array}{l}\text { Scrolling } n(\% \\
N=273)\end{array}$ & $\begin{array}{l}\text { Paging } n(\% \\
N=257)\end{array}$ \\
\hline \multirow[t]{3}{*}{ Recruitment Setting } & General Practice & $59(21.61 \%)$ & $56(21.79 \%)$ \\
\hline & Community & $14(5.13 \%)$ & $12(4.67 \%)$ \\
\hline & Hospital & $200(73.26 \%)$ & 189(73.54\%) \\
\hline \multirow[t]{5}{*}{ Age group } & 18 - 22 years & $15(5.49 \%)$ & $11(4.28 \%)$ \\
\hline & $23-27$ years & $51(18.68 \%)$ & $34(13.23 \%)$ \\
\hline & 28 - 32 years & $90(32.97 \%)$ & $108(42.02 \%)$ \\
\hline & 33 - 37 years & $83(30.40 \%)$ & $81(31.52 \%)$ \\
\hline & $38+$ years & $34(12.45 \%)$ & $23(8.95 \%)$ \\
\hline \multirow[t]{6}{*}{ Ethnic Background } & White & 173(63.37\%) & $182(70.82 \%)$ \\
\hline & Mixed & $9(3.30 \%)$ & $7(2.72 \%)$ \\
\hline & Asian & $64(23.44 \%)$ & $45(17.51 \%)$ \\
\hline & Black & $14(5.13 \%)$ & $14(5.45 \%)$ \\
\hline & Other ethnic group & $12(4.40 \%)$ & $9(3.50 \%)$ \\
\hline & Not disclosed & $1(0.37 \%)$ & $0(0.00 \%)$ \\
\hline \multirow[t]{5}{*}{ Relationship status } & Single & $17(6.23 \%)$ & 13(5.06\%) \\
\hline & $\begin{array}{l}\text { Married/Civil } \\
\text { Partnership }\end{array}$ & 180(65.93\%) & $168(65.37 \%)$ \\
\hline & Cohabiting & $71(26.01 \%)$ & $67(26.07 \%)$ \\
\hline & $\begin{array}{l}\text { Divorced/ } \\
\text { Widowed/ } \\
\text { Separated }\end{array}$ & $2(0.73 \%)$ & $3(1.17 \%)$ \\
\hline & Not disclosed & $3(1.10 \%)$ & $6(2.33 \%)$ \\
\hline \multirow[t]{6}{*}{ Employment status } & $\begin{array}{l}\text { Full-time } \\
\text { employment }\end{array}$ & 135(49.45\%) & $141(54.86 \%)$ \\
\hline & $\begin{array}{l}\text { Part-time } \\
\text { employment }\end{array}$ & $52(19.05 \%)$ & $49(19.07 \%)$ \\
\hline & Self-employed & $25(9.16 \%)$ & 19(7.39\%) \\
\hline & $\begin{array}{l}\text { Seeking } \\
\text { employment }\end{array}$ & $6(2.20 \%)$ & 13(5.06\%) \\
\hline & Unemployed & 49(17.95\%) & $34(13.23)$ \\
\hline & Disability & $6(2.20 \%)$ & $1(0.39)$ \\
\hline \multirow[t]{5}{*}{$\begin{array}{l}\text { Highest educational } \\
\text { achievement }\end{array}$} & $\begin{array}{l}\text { Postgraduate } \\
\text { degree (MSc, PhD) }\end{array}$ & $56(20.51 \%)$ & $52(20.23 \%)$ \\
\hline & University degree & 107(39.19\%) & $94(36.58 \%)$ \\
\hline & $\begin{array}{l}\text { College degree or } \\
\text { below }\end{array}$ & $95(34.80 \%)$ & $97(37.74 \%)$ \\
\hline & None & $8(2.93 \%)$ & $5(1.95 \%)$ \\
\hline & Other qualification & $7(2.56 \%)$ & $9(3.50 \%)$ \\
\hline
\end{tabular}

clinical depression. Particularly, since we did not conduct diagnostic procedures. However, either possibility should be explored further to avoid any risks of missing mothers-to-be who might have met diagnostic criteria for major depression due to changes in the layout of antenatal depression screening scales. Alternatively, these findings could represent a baseline imbalance in
Table 2 Number of participants according to their answers to the Whooley questions and by survey layout (i.e., Scrolling vs. Paging)

\begin{tabular}{lll}
\hline & $\begin{array}{l}\text { Yes to one or both questions } \\
\text { n(row \%) }\end{array}$ & $\begin{array}{l}\text { No to both questions } \\
\text { n(row \%) }\end{array}$ \\
\hline $\begin{array}{l}\text { Scrolling } \\
N=273\end{array}$ & $62(22.71 \%)$ & $211(77.29 \%)$ \\
$\begin{array}{l}\text { Paging } \\
N=257\end{array}$ & $60(23.35 \%)$ & $197(76.65 \%)$ \\
$\begin{array}{l}\text { Total } \\
N=530\end{array}$ & $122(23.02 \%)$ & $408(76.98 \%)$ \\
\hline
\end{tabular}

another factor not captured by our baseline survey, or an increased number of false positives.

\section{Completion time and participants' request for technical assistance}

In this study, we chose completion time and participants' requests for technical assistance as indicators of the potential added burden of mobile-based depression screening on the workload and schedules of clinical staff.

With regard to completion time, most participants (75\%) completed the surveys under $7 \mathrm{~min} 16 \mathrm{~s}$. This measure however, included the socio-demographic survey (which is not a component of depression screening) and is an overestimation of the real duration of the screening process. Therefore, we considered the observed completion times in relation to our internal trials of the Whooley questions and the EPDS alone (i.e., 2 min $28 \mathrm{~s}$ ). This suggests that the implementation of this type of depression screening might cause minimal disruption to a clinics' schedules. Furthermore, we found that participants in the Scrolling group were significantly faster than participants in the Paging group. This difference of $47.5 \mathrm{~s}$ may not be meaningful given the speed of completion.

Approximately $7 \%$ of all participants requested technical assistance during survey completion, which amounted to 35 unique requests (as no participant made more than one request). We found that the choice of survey layout did not affect the proportion of participants asking for help in each group. About $71 \%$ of these requests (25 of 35 ) were to clarify the meaning of questions. The remaining 10 requests were related to

Table 3 Overall mean and median EPDS scores for all participants and by survey layout

\begin{tabular}{lll}
\hline & Mean EPDS score (SD) & Median EPDS score \\
\hline $\begin{array}{l}\text { Scrolling } \\
N=273\end{array}$ & $4.72(4.82)$ & 3 \\
Paging & $4.43(3.80)$ & 3 \\
$N=257$ & & \\
Overall & $4.58(4.35)$ & 3 \\
$N=530$ & & \\
\hline
\end{tabular}


Table 4 Overall number of participants at each EPDS interval and by survey layout

\begin{tabular}{llll}
\hline & $\begin{array}{l}\text { Overall scores } 0-9 \text { points } \\
n(\text { row \%) }\end{array}$ & $\begin{array}{l}\text { Overall scores } 10-12 \text { points } \\
n(\text { row \%) }\end{array}$ & $\begin{array}{l}\text { Overall scores 13+ points } \\
n(\text { row \%) }\end{array}$ \\
\hline $\begin{array}{l}\text { Scrolling } \\
N=273\end{array}$ & $236(86.45 \%)$ & $14(5.13 \%)$ & $23(8.42 \%)$ \\
$\begin{array}{l}\text { Paging } \\
N=257\end{array}$ & $225(87.55 \%)$ & $24(9.34 \%)$ & $8(3.11 \%)$ \\
$\begin{array}{l}\text { Total } \\
N=530\end{array}$ & $461(86.98 \%)$ & $38(7.17 \%)$ & $31(5.85 \%)$
\end{tabular}

difficulties navigating through the surveys (due to the implementation of validation rules that prevented participants from progressing through the surveys leaving questions unanswered) and interacting with the user interface (namely, interacting with the response options). These findings suggest that this population is already familiar with mobile technology, also implied by the level of smartphone and tablet ownership in our sample (approximately $98 \%$ and $82 \%$, respectively), and that there is no need for clinical members of staff to oversee the survey completion process. Instead, these requests could be dealt with by non-clinical support staff (as long as patient have the opportunity to discuss their screening results with a clinician during their appointment).

\section{Deployment challenges identified during the feasibility study}

A perceived concern that has been reported in the literature is the location and privacy of screening $[5,22]$. This concern was also voiced by many clinicians at the various recruitment sites prior initiating the study. We chose recruitment and completion rates as indicators of whether participants felt comfortable undergoing depression screening in the waiting area of antenatal clinics. We found that most eligible mothers-to-be who were approached (approximately 90\%) agreed to take part in the study, and approximately $91 \%$ of those who took part completed the study procedures. In those sites where the research midwives or CSOs recorded the reasons given by eligible mothers-to-be for refusing to take part in the study, we found that they expressed a lack of interest in the study and not wanting to miss being called to their appointment. Moreover, the only reason that some participants could not complete the study procedures (i.e., breakoff) was that they were called in to

Table 5 Number of participants by score on question 10 of the EPDS and by survey layout

\begin{tabular}{lll}
\hline & $\begin{array}{l}0 \text { points on question } 10 \\
n(\text { row \%) }\end{array}$ & $\begin{array}{l}1 \text { point or more on question } 10 \\
n(\text { row } \%)\end{array}$ \\
\hline $\begin{array}{l}\text { Scrolling } \\
N=273\end{array}$ & $268(98.17 \%)$ & $5(1.83 \%)$ \\
$\begin{array}{l}\text { Paging } \\
N=257\end{array}$ & $251(97.67 \%)$ & $6(2.33 \%)$ \\
\hline
\end{tabular}

their appointment. These findings suggest that mobile technology may offer sufficient privacy and anonymity to patients, so that they are able to complete depression screening scales in a clinic's waiting area before their antenatal appointment.

We initially intended to use breakoff rates (and the corresponding reasons) as an indicator of participants' perceived intrusiveness of the screening process. Since participants suspended their participation only if they were called in to their appointment, this outcome became an indicator of the compatibility between clinical workflows and research procedures. Overall, 133 women were called in to their appointment at different stages of the study: 44 (33\%) before being approached by a research midwife or CSO, 35 (26\%) before they were able to complete the consent process, and $54(41 \%)$ before they were able to complete the study procedures.

The main difficulty during the initial stage was researchers' lack of time or capacity. Most women called in before being approached about participation (37 of 44 or approximately $84 \%$ ) were in primary care facilities with short waiting times between patient check in and appointments. This presented a challenge for research midwives and CSOs working in these facilities, as they struggled to recruit enough participants into the study. The remaining women (7 of 44 or approximately 16\%) were in secondary care sites with a larger volume of patients and longer waiting times. In these facilities however, research midwives and CSOs did not have the capacity to approach all the eligible mothers-to-be who were identified.

Although most women who were called in during the consent process or the study procedures were in

Table 6 Number of participants by requests for technical assistance and by experimental group

\begin{tabular}{lll}
\hline & $\begin{array}{l}\text { No requests } \\
\mathrm{n}(\text { row \%) }\end{array}$ & $\begin{array}{l}\text { Requests for technical assistance } \\
\mathrm{n}(\text { row \%) }\end{array}$ \\
\hline $\begin{array}{l}\text { Scrolling } \\
N=273\end{array}$ & $254(93.04 \%)$ & $19(6.96 \%)$ \\
$\begin{array}{l}\text { Paging } \\
N=257\end{array}$ & $241(93.77 \%)$ & $16(6.23 \%)$ \\
$\begin{array}{l}\text { Total } \\
N=530\end{array}$ & 495(93.40\%) & $35(6.60 \%)$ \\
\hline
\end{tabular}


Table 7 Types of requests for technical assistance by experimental group

\begin{tabular}{llll}
\hline Type of request & $\begin{array}{l}\text { Scrolling - number of requests (\% of } 19 \\
\text { requests) }\end{array}$ & $\begin{array}{l}\text { Paging - number of requests (\% of 16 } \\
\text { requests) }\end{array}$ & $\begin{array}{l}\text { Row total (\% of } 35 \text { requests across both } \\
\text { groups) }\end{array}$ \\
\hline $\begin{array}{l}\text { Clarification of } \\
\text { question }\end{array}$ & $14(73.68 \%)$ & $11(68.75 \%)$ & $25(71.43 \%)$ \\
Interface issues & $1(5.26 \%)$ & $1(6.25 \%)$ & $2(5.71 \%)$ \\
Navigation issues & $0(0 \%)$ & $3(18.75 \%)$ & $3(8.57 \%)$ \\
Not recorded & $4(21.05 \%)$ & $1(6.25 \%)$ & $5(14.29 \%)$ \\
\hline
\end{tabular}

secondary care facilities (73 of $89-82 \%$ ), the main difficulty during these stages was the incompatibility between the administrative research procedures and clinical workflows. We have already discussed how the socio-demographic survey (which clinical care teams would not need to administer as they should have access to this information) inflated the real duration of the screening process. However, we also need to consider the additional time required to conduct non-routine, non-clinical procedures and their impact on clinical workflows: providing information about the study, discussing a participant information leaflet, answering any questions that potential participants might have, and completing an informed consent form.

These findings highlight the tension between the need for sound evidence about the impact of technology on antenatal depression screening, and the challenges associated with introducing these technologies in clinical settings. They also highlight that the planning of antenatal depression screening through mobile devices must be sensitive to local clinical workflows, and to the role of the staff members (both clinical and non-clinical) who will be responsible for their implementation.

The introduction of mobile technology for antenatal depression screening in clinical settings also needs to take into consideration issues of connectivity. Wi-Fi access varied between the participating clinics. This was a key determinant for our choice of app, as we needed support for offline data collection. We suspect that this might also represent a requirement for clinical teams wishing to implement this type of depression screening. In this context however, there is an added issue concerning the timely transmission of patient results to clinicians. In our study, this was done through a paper form that research midwives and CSOs filled in as soon as participants completed the study. This would introduce an extra administrative step to the routine activities of a clinic, not taking full advantage of the internet capabilities of mobile devices to facilitate data management. Timely transmission of patient results could be a problem without interoperability between the mobile app and clinical systems.

Lastly, the introduction of mobile technology in clinical settings also needs to take into account the management of technology. Security is a key concern in this context, particularly in relation to lost devices or theft. In addition to the economic implications, this could also lead to a breach of confidentiality, if patient data are held on the devices. Therefore, clinical teams should ensure compliance with their organisation's information governance policies without affecting patient experience. For example, passwords are considered ideal for protecting sensitive data. However, passwords could also disrupt clinical workflows if patients using password-protected devices time out and have to request help from staff members. Clinical teams also need to ensure that data are stored and transmitted using approved data encryption standards. Moreover, those responsible for the devices must be aware of any software update containing security patches.

\section{Limitations}

We recruited adult participants who felt comfortable reading and writing in English. However, important risk factors for antenatal depression include young age and individuals from minority groups. We relied on self-disclosure when assessing the eligibility of potential participants. We did not perform diagnostic interviews to assess a diagnosis of depressive disorder, and this limits our ability to determine if there was a true survey layout effect on participants' responses to the depression screening scales. Our measures of completion time included the sociodemographic baseline survey, which is not a component of antenatal depression screening. Therefore, the inclusion of this survey inflated the true completion times. We considered indirect measures of the impact of mobile technology on the activities of antenatal clinics. In some sites, the study procedures were administered by research staff with no links to the clinical team, or by research midwives with dual responsibilities: clinical and research. In addition, the administrative research requirements were unnatural to the reality of antenatal clinics and, in some cases, caused disruption.

\section{Conclusions}

It is feasible to use tablet computers for the administration of validated screening depression scales in the waiting area of antenatal clinics. Survey layout did not 
influence the proportion of mothers-to-be that screen positive using the Whooley questions or their EPDS scores in this study. However, we observed a significant relationship between survey layout and EPDS scoring intervals. This association should be explored further to determine if it corresponds to a survey layout effect, in order to ensure that clinically depressed mothers-to-be are not missed due to non-clinical effects. The use of mobile technology for antenatal depression screening does not seem to disrupt the routine activities of antenatal clinics. Nonetheless, it is crucial that practitioners and commissioners consider the successful integration of this technology into existing clinical workflows, the timing of appointment, as well as connectivity and security issues. These findings are relevant in the context of the design and implementation factors that could affect the quality of the responses given to validated depression screening scales.

\section{Additional files}

Additional file 1: Socio-demographic survey. Description of data: eleven-question survey used to gather socio-demographic information from participants. (DOCX 66 kb)

Additional file 2: Whooley Questions. Description of data: two-item survey used to evaluate the presence of depressive mood and anhedonia over the past month. (DOCX $46 \mathrm{~kb}$ )

Additional file 3: Edinburgh Postnatal Depression Scale. Description of data: a ten-item instrument used to screen for antenatal or postnatal depression in community and clinical settings. This instrument assesses feelings of guilt, sleep disturbance, anhedonia and thoughts of self-harm that have been present for the past 7 days. (DOCX $84 \mathrm{~kb}$ )

\section{Abbreviations}

CSO: Clinical studies office; EPDS: Edinburgh postnatal depression scale; IOS: iPhone operating system; NHS: National health service; NICE: The National Institute for health and care excellence; PIL: Participant information leaflet; SD: Standard deviation

\section{Acknowledgments}

We wish to thank all our participants. We would also like to thank Yvonne Tul from CRN South London; Laura Pearse and Geraldine Landers from Hillingdon Hospital; Joanna Girling and Marie O'Connell from West Middelsex Hospital; Cathie Melvin from East Lancashire Hospitals; Tara Pauley and Charlotte Clayton from Hinchingbrooke Hospital; Anna Fleming from University Hospitals of North Midlands; Jane Radford and Anne Hogg from Burton Hospitals; Angela Loughlin from Shrewsbury and Telford Hospital; Mark Johnson, Roshni Patel, Carmella Martella and Minna Meritahti from Chelsea and Westminster Hospital; Claire Fairhurst and Tracey Taylor from Wrightington, Wigan and Leigh Hospital; Rachel Newport and Zainab Sarwar Yasin from the Pennine Acute Hospitals; Alison Loftus from the Royal Bolton Hospital; Sally Dunn, Siobhan Limerick, and Helen Riding from North East and North Cumbria CRN; Justine Norman from North Tyneside CCG; and Helen Ginelly and Mary Day from East Midlands CRN. Lastly, thank you to Antoinette McNulty, Gemma Loebenberg, Sophie Hannay, Saloni Dosani, Shilpi Mehra and Tara Harvey from CRN Northwest London.

\section{Funding}

This study was funded by the National Institute for Health Research (NIHR) Imperial Biomedical Research Centre (BRC) through its Population Health theme. This study was adopted by the NIHR portfolio under the Primary Care and Mental Health clinical specialties. Therefore, this study received support from the NIHR Comprehensive Research Networks (CRN), with Northwest London as the lead network.

\section{Availability of data and materials}

The datasets used and/or analysed during the current study are available from the corresponding author on reasonable request.

\section{Authors' contribution}

JMB conceived the study, designed the study protocol, acted as the study coordinator, analysed and interpreted the data, and drafted the manuscript. AKG performed the initial sample size calculation, and provided advice on the statistical analysis. PR provided advice on the clinical relevance of the findings. CM, JOD and JC have supervised the work of JMB, and provided guidance and intellectual input. All the authors reviewed and approved the final manuscript.

\section{Authors' information}

This study was conducted as part of the doctoral work of JMB, and will form part of his doctoral thesis.

\section{Competing interests}

The authors declare that they have no competing interests. There is no financial relationship between the authors and Snap ${ }^{\oplus}$ surveys.

\section{Consent for publication}

Not applicable as we did not report individual participant data.

\section{Ethics approval and consent to participate}

This study received favourable opinion from the National Research Ethics Committee South East Coast - Surrey on 09 July 2015 (Reference: 15/LO/ 0977). We obtained local approval from each participating NHS organisation. The research midwives and CSOs recruiting participants into this study had received Good Clinical Practice training, as required in the NHS. All participants were asked to complete an informed consent form before completing the study procedures.

\section{Publisher's Note}

Springer Nature remains neutral with regard to jurisdictional claims in published maps and institutional affiliations.

\section{Author details}

${ }^{1}$ Global eHealth Unit, Department of Primary Care and Public Health, School of Public Health, Imperial College London, London, UK. 'Faculty of Medicine, National Heart and Lung Institute, Imperial College London, London, UK. ${ }^{3}$ Global eHealth Unit, Department of Primary Care and Public Health, School of Public Health, Imperial College London, London, UK. ${ }^{4}$ The Centre for Pyschiatry, Department of Medicine, Imperial College London, London, UK. ${ }^{5}$ Global eHealth Unit, Department of Primary Care and Public Health, School of Public Health, Imperial College London, London, UK. ${ }^{6} \mathrm{Global}$ eHealth Unit, Department of Primary Care and Public Health, School of Public Health, Imperial College London, London, UK.

Received: 18 December 2016 Accepted: 3 May 2017

Published online: 10 May 2017

References

1. BinDhim NF, Shaman AM, Trevena L, Basyouni MH, Pont LG, Alhawassi TM. Depression screening via a smartphone app: cross-country user characteristics and feasibility. J Am Med Inform Assoc 2015; doi: 10.1136/ amiajnl-2014-002840.

2. BinDhim NF, Alanazi EM, Aljadhey H, Basyouni MH, Kowalski SR, Pont LG, Shaman AM, Trevena L, Alhawassi TM. Does a Mobile Phone DepressionScreening App Motivate Mobile Phone Users With High Depressive Symptoms to Seek a Health Care Professional's Help? J Med Internet Res 2016; doi: 10.2196/jmir.5726.

3. Birney AJ, Gunn R, Russell JK, Ary DV. MoodHacker Mobile Web App With Email for Adults to Self-Manage Mild-to-Moderate Depression: Randomized Controlled Trial. JMIR Mhealth Uhealth 2016; doi:10.2196/mhealth.4231.

4. Faurholt-Jepsen M, Frost M, Vinberg M, Christensen EM, Bardram JE, Kessing LV. Smartphone data as objective measures of bipolar disorder symptoms. Psychiatry Res 2014; doi:10.1016/j.psychres.2014.03.009.

5. Tabb KM, Choi S, Pineros-Leano M, Meline B, McDonald HG, Kester R, Huang H. Perinatal depression screening in a Women, Infants, and Children (WIC) program: perception of feasibility and acceptability among a 
multidisciplinary staff. Gene Hosp Psychiatry 2015; doi:10.1016/j. genhosppsych.2015.03.008.

6. Marcano-Belisario JS, Jamsek J, Huckvale K, O'Donoghue J, Morrison CP, Car J. Comparison of self-administered survey questionnaire responses collected using mobile apps versus other methods. Cochrane Database Syst Rev 2015; doi:10.1002/14651858.MR000042.pub2.

7. Gavin NI, Gaynes BN, Lohr KN, Maltzer-Brody S, Gartlehner G, Swinson T. Perinatal depression: a systematic review of prevalence and incidence. Obstet Gynecol. 2005;106(5 Pt 1):1071-83.

8. Bennett HA, Einarson A, Taddio A, Koren G, Einarson TR. Prevalence of depression during pregnancy: systematic review. Obstet Gynecol. 2004; 103(4):698-709.

9. Stewart DE. Clinical practice: Depression during pregnancy. N Engl J Med 2011; doi:10.1056/NEJMcp1 102730.

10. Heron J, O'Connor TG, Evans J, et al. The course of anxiety and depression through pregnancy and the postpartum in a community sample. J Affect Disord 2004; doi:10.1016/j.jad.2003.08.004

11. Kinsella MT, Monk C. Impact of Maternal Stress, Depression \& Anxiety on Fetal Neurobehavioral Development. Clin Obstet Gynecol 2009;doi:10.1097/ GRF.0b013e3181b52df1.

12. Pearson RM, Evans J, Kounali D, et al. Maternal Depression During Pregnancy and the Postnatal Period: Risks and Possible Mechanisms for Offspring Depression at Age 18 Years. JAMA Psychiatry 2013; doi:10.1001/ jamapsychiatry.2013.2163

13. Braithwaite EC, Murphy SE, Ramchandani PG. Effects of prenatal depressive symptoms on maternal and infant cortisol reactivity. Arch Womens Ment Health 2016;doi:10.1007/s00737-016-0611-y.

14. Bauer A, Parsonage M, Knapp M, lemmi V, Adelaja B. The costs of perinatal mental health problems. London: Centre for Mental Health and London School of Economics; 2014

15. Woolhouse H, Gartland D, Mensah F, Brown SJ. Maternal depression from early pregnancy to 4 years postpartum in a prospective pregnancy cohort study: implications for primary health care. BJOG 2015; doi:10.1111/1471-0528.12837.

16. National Collaborating Centre for Mental Health. Antenatal and postnatal mental health: clinical management and service guidance-updated edition. NICE guideline (CG192). Leicester London: British Psychological Society and The Royal College of Psychiatrists; 2014.

17. Sherwin HN, McKeown M, Evans MF, Battacharyya OK. The waiting room "wait": from annoyance to opportunity. Can Fam Physician. 2013;59(5):479-81.

18. Mavletova A, Couper M. Mobile web survey design: scrolling versus paging, SMS versus e-mail invitations. J Surv Stat Methodol. 2014;2:498-518.

19. Wells T, Bailey JT, Link MW. Comparison of smartphone and online computer survey administration. Soc Sci Comput Rev. 2014;32:238-55.

20. Keusch F. The Influence of Answer Box Format on Response Behaviour on List-Style Open-Ended Questions. J Surv Stat Methodol 2014; doi:10.1093/ jssam/smu007.

21. Pineros-Leano M, Tabb KM, Sears H, Meline B, Huang H. Clinic staff attitudes towards the use of mHealth technology to conduct perinatal depression screenings: a qualitative study. Fam Pract 2015; doi:10.1093/fampra/cmu083.

22. Marcano-Belisario JS, Gupta AK, O'Donoghue J, Morrison C, Car J. Tablet computers for implementing NICE antenatal mental health guidelines: protocol of a feasibility study. BMJ Open 2016; doi:10. 1136/bmjopen-2015-009930.

23. Snap Surveys Limited. Powerful and flexible software. [Online] Available at: https://www.snapsurveys.com/survey-software/. Accessed 12 Sept 2016.

24. Snap Surveys Limited. Snap Mobile. [Online] Available at: https://itunes.apple. com/gb/app/snap-mobile/id507440809?mt=8. Accessed 12 Sept 2016.

25. Snap ${ }^{\oplus}$ Surveys Limited. Snap WebHost - Online and Mobile Survey Management. [Online] Available at: https://www.snapsurveys.com/surveysoftware/snap-webhost-online-mobile-survey-management-system/. Accessed 12 Sept 2016.

26. Whooley MA, Avins AL, Miranda J, Browner WS. Case-finding instruments for depression - two questions are as good as many. J Gen Intern Med. 1997;12: 439-45.

27. Cox JL, Holden JM, Sagovsky R. Detection of postnatal depression development of the 10-item Edinburgh Postnatal Depression Scale. Br J Psychiatry. 1987;150:782-6.

28. Allbaugh $\sqcup$, Marcus SM, Ford EC, Flynn HA. Development of a screening and recruitment registry to facilitate perinatal depression research in obstetrics settings in the USA. Int J Gynaecol Obstet 2015; doi: 10.1016/j. ijgo.2014.09.015

29. Stata. Stata Statistical Software [Program]. Version 13, 2013

30. Core Team R. A language and environment for statistical computing. Vienna, Austria: R Foundation for Statistical Computing; 2016. https://www R-project.org/.

\section{Submit your next manuscript to BioMed Central and we will help you at every step:}

- We accept pre-submission inquiries

- Our selector tool helps you to find the most relevant journal

- We provide round the clock customer support

- Convenient online submission

- Thorough peer review

- Inclusion in PubMed and all major indexing services

- Maximum visibility for your research

Submit your manuscript at www.biomedcentral.com/submit
) Biomed Central 